\title{
44591 - POSTOPERATIVE MI AFTER NON CARDIAC SURGERY IN PATIENTS WITH PREVIOUS PCI
}

\author{
Adriaan Van Rensburg, Toronto General Hospital, University Health Network, Toronto, \\ ON, Canada; \\ Duminda Wijeysundera, Toronto General Hospital, University Health Network; \\ Massimiliano Meineri, Toronto General Hospital, University Health Network; \\ M Wasowicz, Toronto General Hospital, University Health Network; \\ VDzavik; \\ WS Beattie, Toronto General Hospital, University Health Network;
}

INTRODUCTION: Patients having non-cardiac surgery after Percutaneous Coronary Interventions (PCI) have increased morbidity and morality. Multiple risk factors for complications have been previously identified, including a short interval from the time of PCI to surgery, lack of anti-platelet drugs, and the use of intra operative heparin. The role of Drug Eluting Stents (DES) compared to bare Metal Stent in the increased morbidity is unknown. The purpose of this investigation was to assess these multiple factors on post operative myocardial infarction (POMI) and Death.

METHODS: After REB approval, the hospital identification number of all patients having noncardiac surgery in our institution from April 2003 to June 2006 was compared with our cardiology stent data base. We thereby identified patients having non-cardiac surgery after PCI. Post operative myocardial infarction (troponin I $>0.07 \mathrm{ng} / \mathrm{ml}$ with associated symptoms or EKG changes) was the dependant variable. The unadjusted relationship between lag time between stent deployment, the type of stent, medications, transfusion, and postoperative cardiac complications were determined using appropriate tests (t-test, Mann-Whitney U test, $\mathrm{c} 2$ test, Fisher's exact test). variables with a $\mathrm{p}<0.1$ were considered as potential confounders. Backward stepwise variable selection was employed to construct a regression model. The association of independent predictors with MI was expressed as odds ratios (OR, 95\% CI).Statistical analyses were performed using SAS Version 8.20 software (SAS Institute, Cary, NC, USA). Statistical significance was defined by $\mathrm{P} £ 0.05$.

RESULTS: 120 patients were identified as having surgery after PCI. $85 \%$ of patients have antiplatelet therapy continued throughout the perioperative period. $80 \%$ were continued on beta blockers, $76 \%$ were continued on statins. There were 21 perioperative MI's (17.\%) Lag time, the use of intra-operative heparin and transfusion of red blood cells were found to be independent predictors of postoperative MI .Time from deployment OR $3.6(1.1-12.5) \mathrm{p}=.045$,Intra operative heparin OR 5.1(1.6-15.7)p=.007, Transfusion> 1 unit RBC OR 8.2 (1.9-35)p=.005.

Figure 1 shows the relationship between lag between deployment of the stent and surgery and perioperative MI. We could not demonstrate that DES compared to BMS increased the risk of POMI

CONCLUSION: The rate of POMI after PCI is in excess of $35 \%$ immediately after deployment and declines in a linear manner until the risk of POMI plateaus at 12 months. The rate of 
perioperative MI after 12 months (11.5\%) is the same as patients with a Revised Cardiac Risk Index class III. Intraoperative heparin, and transfusion are independent predictors of MI after PCI. After PCI patients must be considered high risk.

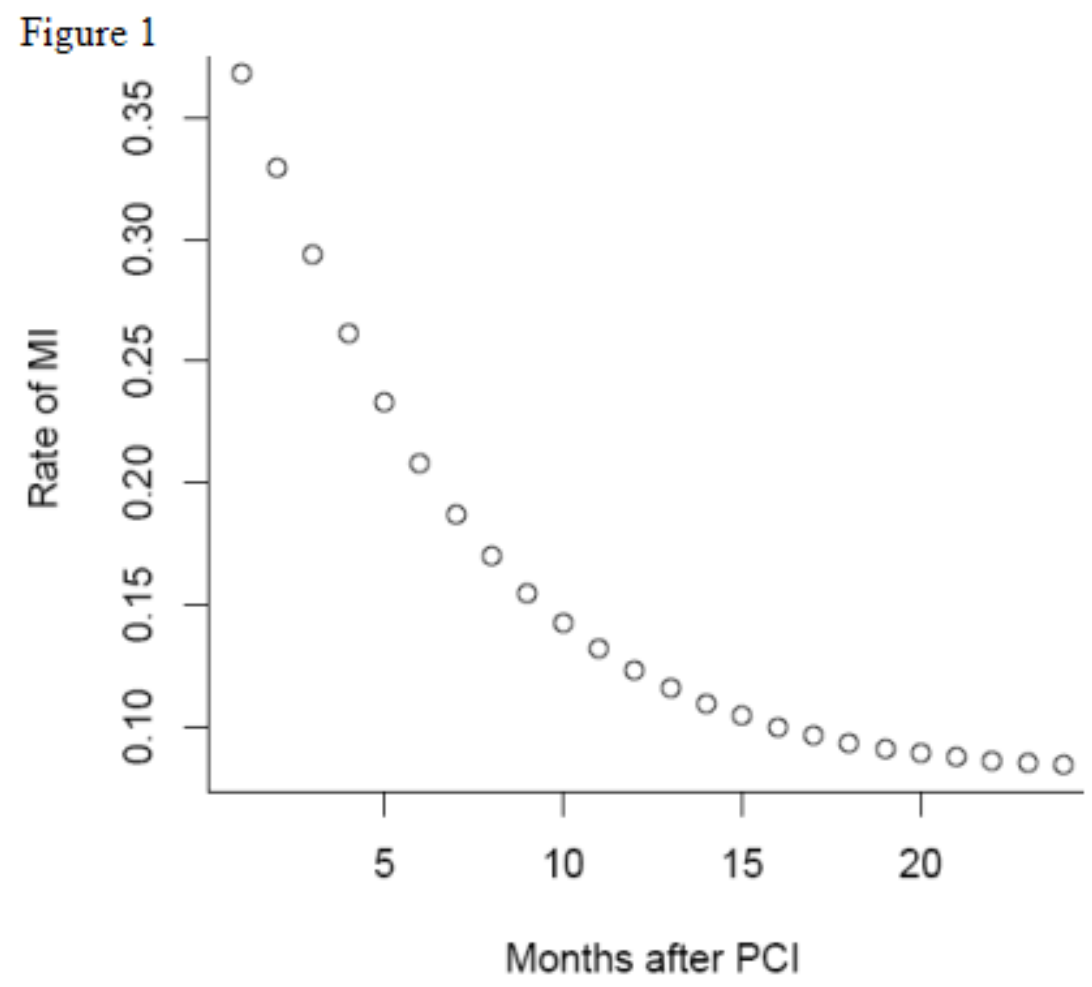

The relationship between time of PCI to surgery on the rate of POMI after non-cardiac surgery. 